\title{
Groin Hernia Surgery in Uganda: Caseloads and Practices at Hospitals Operating Within the Publicly Funded Healthcare Sector
}

\author{
Alphonsus Matovu ${ }^{1,2} \cdot$ Pär Nordin ${ }^{3} \cdot$ Andreas Wladis $^{4} \cdot$ Mary Margaret Ajiko $^{5} \cdot$ \\ Jenny Löfgren ${ }^{5}$
}

Published online: 15 June 2020

(c) The Author(s)

\begin{abstract}
Background Groin hernia is a major public health problem with over 200 million people affected. The unmet need for surgery is greatest in Sub-Saharan Africa where specialist surgeons are few. This study was carried out in Uganda to investigate caseloads and practices of groin hernia surgery at publicly funded hospitals.

Methods The study employed mixed methods covering 29 hospitals: the National Referral Hospital (NRH), 14 Regional Referral Hospitals (RRH) and 14 General Hospitals (GH). In part one of the study, surgeons and medical doctors performing hernia repair were interviewed about their practices and experiences of groin hernia surgery. In part two, operating theater records from 2013 to 2014 from the participating hospitals were reviewed and information about groin hernia operations collected.

Results All respondents reported that sutured repair was the first-choice method. A total of 5518 groin hernia repairs were performed at the participating hospitals, i.e., an annual hernia repair rate of 7/100 000 population. Of the patients operated, almost $16 \%$ were women and $24 \%$ were children. Local anesthesia (LA) was used in $40 \%$ of the cases, and non-surgeon physicians performed $70.3 \%$ of the groin hernia repairs.

Conclusion Groin hernia repair outputs need to increase along with the training of surgical providers in modern hernia repair methods. Methods and outcomes for hernia repair in women and children should be investigated to improve the quality of care.
\end{abstract}

Alphonsus Matovu

alphonsusing@gmail.com

1 Department of Molecular Medicine and Surgery, Karolinska Institutet, Karolinska University Hospital, 17176 Stockholm, Sweden

2 Department of Surgery, Mubende Regional Referral Hospital, Plot M.4 Kakumiro Road, P.O Box 4, Mubende, Uganda

3 Department of Surgery and Perioperative Sciences, Umeå University, Umeå, Sweden

4 Department of Clinical and Experimental Medicine, Linköping University, Linköping, Sweden

5 Department of Molecular Medicine and Surgery, Karolinska Institute, Solna, Sweden

\section{Introduction}

Groin hernia repair is the most commonly performed general surgical procedure worldwide [1]. Over 200 million people are affected with groin hernias globally [2], and untreated groin hernias have significant morbidity and mortality [3]. In Sub-Saharan Africa (SSA), the volume of groin hernia surgery relative to the prevalence is insufficient [4-6]. Many people live with untreated groin hernias representing an enormous unmet need for surgery [7, 8]. In Ghana, the annual incidence of symptomatic hernias was $210 / 100,000$ people with an estimated hernia repair rate of 30/100,000 [9]. In Uganda, the total groin hernia prevalence rate was $9.4 \%, 6.6 \%$ untreated or with recurrence and $3.3 \%$ with scars and an annual inguinal hernia repair rate of 
17/100,000 people [7]. In Sierra Leone, of the 93.2\% people who indicated the need for health care, only $22.3 \%$ underwent a surgical procedure [10].

Groin hernia surgery techniques have evolved from stimulating inflammation by cauterization and application of a golden stitch, using a hernia support, narrowing of the hernia orifice, the Bassini and Shouldice suture methods to the current tension-free mesh repair [11-13]. The Shouldice technique is superior to non-mesh based methods; however, it is usually performed by highly trained surgeons at specialized centers [14]. Over time, the use of synthetic mesh is recommended as the first-choice method in inguinal hernia repair, because of less recurrence and postoperative pain [15]. Further potential improvements in groin hernia surgery include use of Local Anesthesia (LA) and day case surgery [16-19]. The extent of mesh application, LA and day case surgery in SSA is not known. This nationwide study from 2013 to 2014 investigated surgical caseloads and practices of groin hernia surgery in Uganda, an East African low-income country of 39 million inhabitants [20].

\section{Materials and methods}

This study was carried out at 29 publicly funded hospitals throughout Uganda (Fig. 1). These were the National Referral Hospital (NRH), all 14 Regional Referral Hospitals (RRH) and 14 General Hospitals (GH) each affiliated to a RRH. The GHs were selected according to convenience. In Uganda, the healthcare system has primary, secondary and tertiary levels. The GH represents the primary level covering a catchment population of approximately 500,000 people. Specialist surgeons and anesthesiologists are normally not employed in these hospitals where basic surgical procedures such as hernia repair and cesarean section are performed. The RRH represents the secondary level of healthcare, constructed for a catchment population of two million people. The services offered include specialized medical and surgical care, and at least one specialist surgeon should be employed. In addition, RRHs train intern doctors who can perform surgical procedures like hernia repair and cesarean section, medical, paramedical and nursing students. They support and receive patients from the GHs within their catchment area. The NRH is the tertiary level of healthcare serving the whole population with subspecialized medical and surgical care, advanced diagnostics, research, and training of medical doctors, nurses and paramedics [21].

The study comprised two parts. Part one was a questionnaire-based interview answered by surgeons and medical doctors performing groin hernia repair at the participating hospitals. The questionnaire was piloted by the lead investigator before using it in the study. From each hospital, a surgically active surgeon or medical officer was interviewed. A surgeon in Uganda is usually a medical doctor who has completed a minimum of 3 years of surgical training following medical school and internship. A medical doctor is a doctor who has completed medical school and internship.

The questionnaire included methods for hernia repair and anesthesia used in elective and emergency groin hernia surgery, duration of hospital stay and practice of day case surgery. Interviews were carried out by the research team after obtaining informed consent from the respondents.

Part two was a retrospective review of data from 2013 to 2014 theater records. These records are routinely kept at all hospitals in Uganda. Record books may be found stored in the operating theater or in the archives. The theater records have information on surgical volume, patient demographics and the procedures performed at any particular hospital. The information includes date of the surgical procedure, gender, age, patient diagnosis, the surgeon's name, the surgical procedure and the form of anesthesia used. Data on groin hernia procedures were extracted from these records and entered into excel spread sheets. Some of the data were missing, and the recovered data were used to cater for missing data by imputation. Microsoft Excel and SPSS were used for descriptive and comparative data analyses.

Ethics approval was obtained from Makerere University School of Public Health Higher Degrees Research and the Ethics Committee and the Uganda National Council of Science and Technology. Prior to data collection, the Medical Superintendent and Hospital Director of each hospital signed a consent form authorizing collection of data. All respondents in part one of the study gave their informed consent prior to the interview.

\section{Results}

Twenty-nine respondents, 16 surgeons and 13 medical doctors, were interviewed (Table 1). Sutured techniques, mainly the modified Bassini, were the methods of choice for all respondents in both elective and emergency groin hernia repair. The reasons mentioned were availability of sutures, affordability and that the technique is well known. The respondents rarely used the mesh technique due to non-availability of the mesh, high costs and lack of possibilities to learn how to perform mesh repair. Its use was limited to teaching and patients who could afford it.

In elective cases, $56.3 \%$ of the specialists and $46.1 \%$ of the medical doctors prefer LA in a small inguinal hernia and day case surgery. The mentioned benefits of using LA were affordability and not requiring anesthetic resources because surgeons administer it themselves. There was no distinction between LA and ilioinguinal nerve block. 
Fig. 1 Geographic distribution of hospitals included in the study

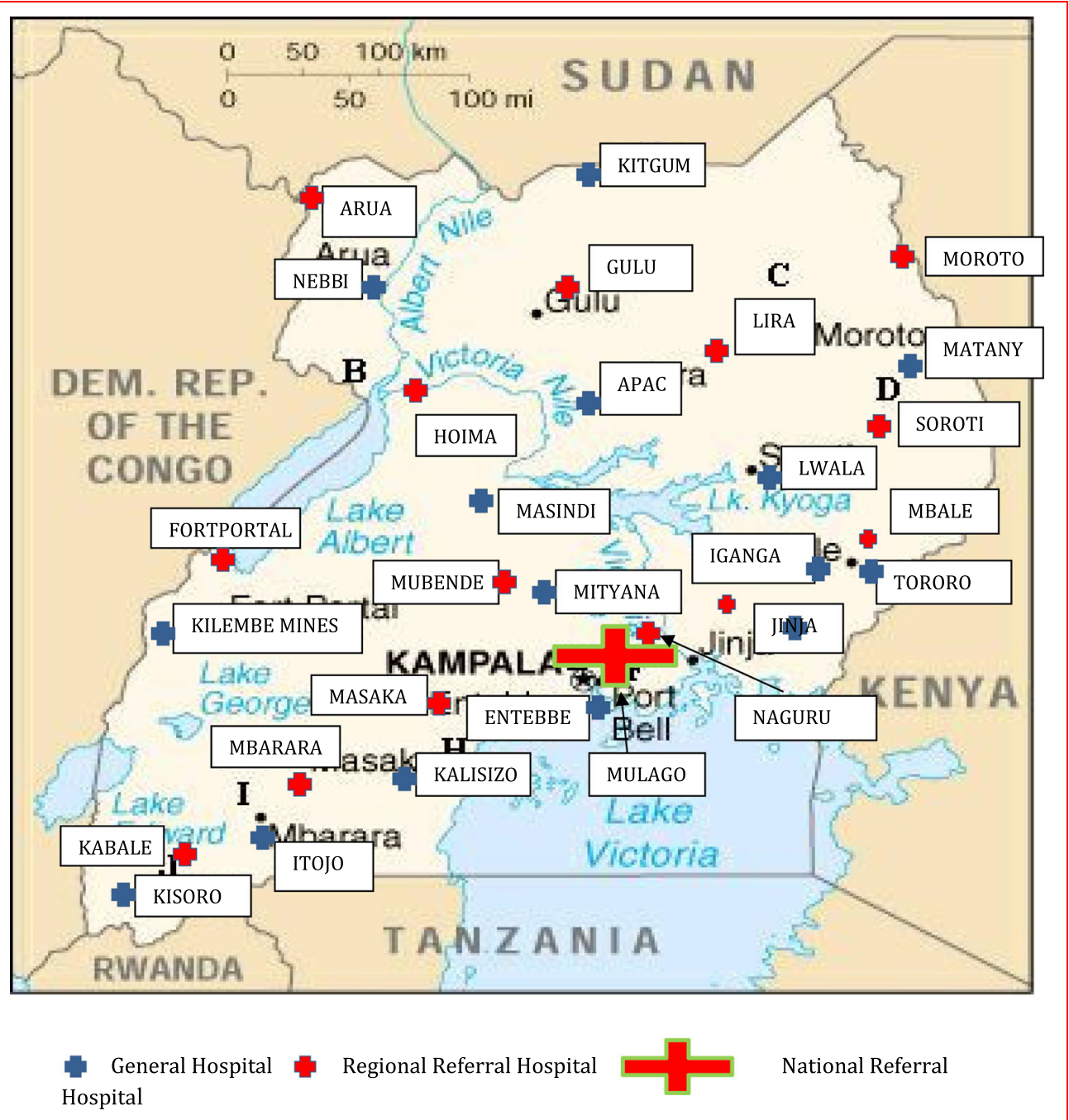

Spinal Anesthesia (SA) was the method of choice for patients with a large inguinoscrotal hernia, for recurrent hernia and when the absence of oxygen prevented the use of General Anesthesia (GA). SA could also be used instead of LA to avoid pain or discomfort associated with LA. GA was preferred by $25 \%$ of the specialists and $7.7 \%$ of the medical doctors mainly for large inguinoscrotal hernias and pediatric groin hernia surgery. Patient preference occasionally determined the choice for anesthesia.

In emergency cases, obstructed or strangulated groin hernia with suspected gangrenous bowel, $68.8 \%$ of the specialists and $53.8 \%$ of the medical doctors preferred GA. SA was the second most common form of anesthesia, especially for obstructed or strangulated groin hernia or when GA was not available. In emergency cases with small obstructed hernia and no suspicion of gangrenous bowel, LA was preferred by two of the respondents.

Day case surgery was practiced in 17 hospitals, mostly due to limited bed space and patient preference. Furthermore, the respondents stated that it is cost-effective for both the hospital and the patients. The other 12 respondents did not practice day case surgery due to the common use of GA or SA and long journeys for patients to return home. The respondents said that some patients preferred to be admitted until all sutures are removed, in other cases the operating doctor preferred to observe patients for at least $24 \mathrm{~h}$ prior to discharge so that early complications could be attended to. Duration of hospital stay varied between less than 1 day to more than 7 days, mainly depending on whether the procedure was performed electively or as an emergency.

Record reviews showed that 5518 groin hernia repairs were performed in the 29 hospitals in 2013 and 2014. Of the patients where gender was reported, women represented $15.9 \%(n=762)$ of the patients and $23.3 \%$ of the patients were below 18 years. The age distribution was biphasic with a peak incidence of hernia repair in the age group under 5 years and a second peak later in life (Fig. 2).

The numbers of hernia repairs in 2013 and 2014 were almost identical: 2765 and 2753, respectively. The 
Table 1 Interviews with key surgical providers in the 29 study hospitals

\begin{tabular}{lcc}
\hline & Specialist $(n=16)^{\mathrm{a}}$ & Medical doctor $(n=13)$ \\
\hline $\begin{array}{l}\text { First choice for surgical technique } \\
\text { Sutured technique }[n(\%)]\end{array}$ & $16(100)$ & $13(100)$ \\
$\begin{array}{l}\text { First-choice form of anesthesia, elective surgery } \\
\text { Local anesthesia }\end{array}$ & $9(56.3)$ & $6(46.1)$ \\
Spinal anesthesia & $3(18.8)$ & $6(46.1)$ \\
General anesthesia & $4(25)$ & $1(7.7)$ \\
First-choice form of anesthesia, emergency surgery & \\
Local anesthesia & $2(12.5)$ & $0(0)$ \\
Spinal anesthesia & $3(18.8)$ & $6(46.2)$ \\
General anesthesia & $11(68.8)$ & $7(53.8)$ \\
Day case surgery & $10(62.5)$ & $8(61.5)$ \\
Yes & $3(18.8)$ & \\
Preferred length of hospital stay after elective & surgery (days) & \\
$<1$ & $10(62.5)$ & $10(76.9)$ \\
$1-3$ & $2(12.5)$ & $1(7.7)$ \\
$4-6$ & $1(6.3)$ & $0(0)$ \\
7 or more &
\end{tabular}

${ }^{\mathrm{a}}$ Fourteen specialist surgeons and two specialist gynecologists

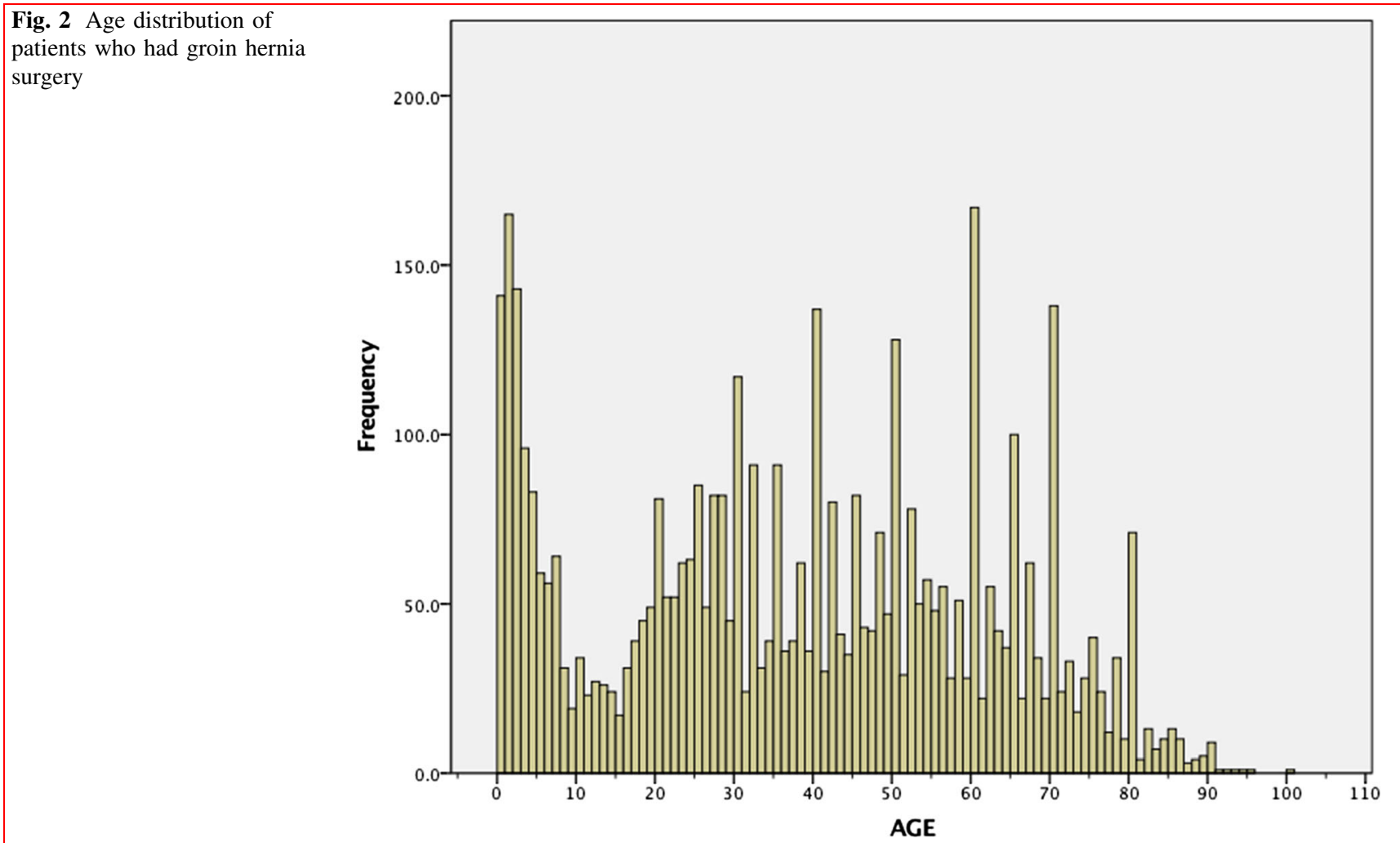

operations were mainly performed at the GH $(n=2430$, $44 \%)$ and the RRH $(n=2711,49.1 \%)$. Only a few procedures were performed at the NRH $(n=377,6.8 \%)$. The
Ugandan population was 39 million in 2014, making the collective surgical volume of the hospitals included in this study 7 hernia repairs per 100,000 population and year. 
Medical doctors performed $2620(50.8 \%)$ of the groin hernia repairs, while intern doctors performed 1006 $(19.5 \%)$. The remaining repairs were performed by specialist surgeons (Table 2). Specialist surgeons together with intern doctors performed the majority of the hernia repairs in the RRHs, whereas medical doctors performed the majority of the repairs in the GHs and the NRH. In the RRHs and the NRH, the surgeon was mostly assisted by another medical doctor, while in the GHs the assistant was usually a nurse.

LA and GA were used to the same extent: $40.0 \%$ and $39.4 \%$, respectively. SA was used in $20.5 \%$. The most common healthcare professional administering GA and SA was the anesthetic officer. These are professionals with a 3-year diploma in clinical medicine, midwifery or nursing in addition to 2 years training in anesthesia. Specialist physician anesthetists administered $4.2 \%$ of the GA and SA.

\section{Discussion}

The results of this study on hernia repair performed within the Ugandan publicly funded healthcare sector reveal that hernia repair is most often performed under local or general anesthesia using sutured techniques. The tertiary and the secondary hospitals together with a selection of GHs perform around 7 groin hernia repairs per 100,000 population and year. The operations are mostly performed by nonspecialist medical doctors. Women represent $16 \%$ of the cases, and almost a quarter are children.

To improve the quality of surgery, hernia repair techniques must comply with international standards. In this study, all respondents use sutured techniques as their first choice. This means that majority of patients may not benefit from the advantages of mesh repair with lower recurrence rates compared to sutured methods [22]. Sutured techniques are preferred to mesh repair because mesh technique is expensive, not known or mastered by the respondents.

Table 2 Level of training of the surgeon, surgical and anesthesia methods at the National Referral Hospital, the Regional Referral Hospitals and the General Hospitals

\begin{tabular}{|c|c|c|c|c|}
\hline & $\mathrm{NRH}[n(\%)]$ & $\mathrm{RRH}[n(\%)]$ & $\mathrm{GH}[n(\%)]$ & $P$ value \\
\hline \multicolumn{5}{|c|}{ Level of training of surgeon, $n=5157$} \\
\hline Specialist surgeon & $52(16.1)$ & $1038(39.4)^{\mathrm{a}}$ & $77(3.6)$ & \multirow[t]{3}{*}{$<0.01$} \\
\hline Medical doctor after internship & $261(81.1)$ & $589(22.4)$ & $2125(96.4)$ & \\
\hline Intern doctor & $9(2.8)$ & $1006(38.2)$ & $0(0)$ & \\
\hline \multicolumn{5}{|c|}{ Level of training of assistant, $n=3511$} \\
\hline Medical doctor after internship & $41(17.8)$ & $216(10.1)$ & $118(10.2)$ & \multirow[t]{5}{*}{$<0.01$} \\
\hline Intern doctor & $169(73.5)$ & $1585(74.4)$ & $0(0)$ & \\
\hline Nurse & $15(6.5)$ & $167(7.8)$ & $700(60.8)$ & \\
\hline Student & $4(1.7)$ & $123(5.8)$ & $37(3.2)$ & \\
\hline Theater assistant & $1(0.4)$ & $38(1.8)$ & $297(25.8)$ & \\
\hline \multicolumn{5}{|c|}{ Level of training of anesthetist giving spinal and general anesthesia, $n=2900$} \\
\hline Specialist anesthesiologist & $38(14.3)$ & $84(6.1)$ & $0(0)$ & \multirow[t]{8}{*}{$<0.01$} \\
\hline Anesthetic officer & $215(80.8)$ & 975 (70.9) & 915 (72.7) & \\
\hline Anesthetic assistant & $1(0.4)$ & $140(10.2)$ & $203(16.1)$ & \\
\hline Surgeon & $2(0.8)$ & $33(2.4)$ & $1(0.1)$ & \\
\hline Medical officer after internship & $1(0.4)$ & $105(7.6)$ & $67(5.3)$ & \\
\hline Intern doctor & $0(0)$ & $30(2.2)$ & $0(0)$ & \\
\hline Nurse & $0(0)$ & $0(0)$ & $14(1.1)$ & \\
\hline Student & $9(3.4)$ & $8(0.6)$ & $59(4.7)$ & \\
\hline \multicolumn{5}{|l|}{ Anesthesia method, $n=5010$} \\
\hline Local anesthesia & $41(13.1)$ & $1106(43.7)$ & 859 (39.6) & \multirow[t]{3}{*}{$<0.01$} \\
\hline Spinal anesthesia & $81(26.0)$ & $511(20.2)$ & $436(20.1)$ & \\
\hline General anesthesia & $190(60.9)$ & $912(36.1)$ & $874(40.3)$ & \\
\hline
\end{tabular}

${ }^{a}$ Including three operations by a gynecologist 
Surgical safety is a particular concern and increased use of LA would be an important step in that direction. LA is recommended for uncomplicated, unilateral, open primary hernia repair [23, 24]. It is safe, effective and more costeffective than SA or GA [25, 26]. In this study, LA was used in $40 \%$ of hernia repairs. This is far higher than the $1.1 \%$ in another SSA setting [27]. It is also far higher than in high-income countries like Sweden where only $13 \%$ of groin hernia repairs are performed under LA. If LA is used, the anesthesia workforce can direct their attention to procedures that require SA or GA.

The groin hernia surgery rate demonstrated in this study is low considering that these hospitals represent the largest public hospitals in the country. Hernia repair has been found to be the most commonly performed general surgical procedure in previous studies in the region $[4,6]$. Still, the prevalence of untreated groin hernia was above $6 \%$ in adult men in a study from Eastern Uganda [6]. Increasing the number of repairs is essential to reduce the burden of disease due to hernia. In Ghana, a country with a large unmet need for groin hernia repair, it is estimated that an operation rate of 420 per 100,000 inhabitants per year would eliminate symptomatic groin hernia within 10 years $[5,9]$. This elimination repair rate was developed by equating the inguinal hernia prevalence, to the symptomatic inguinal hernia incidence, the symptomatic inguinal hernia incidence, surgical repair, the 100-year case backlog and the DALYs associated with backlog in millions [9]. This rate is higher than in western countries (130-290/100,000 population and year) where backlog is minimal $[28,29]$. Our methodology was not set to determine the operation rate that would eliminate the backlog in Uganda, but it would probably require a similar or higher target rate to that in Ghana.

The majority of hernia repairs were performed by medical doctors, while interns performed close to $20 \%$ of all hernia repairs. Task-sharing involves the delegation of certain medical responsibilities to less specialized health care workers [30, 31]. In Uganda, specialist and resident surgeons are few in number and unevenly distributed between urban and rural areas. Basic surgical procedures such as groin hernia repair are therefore frequently performed by non-specialist surgeons. The provision of structured training for intern doctors and non-specialist physicians would increase surgical capacity and to improve the quality of surgical care for hernia in Uganda. In order to build such capacity, hernia repair must be advocated for as a public health intervention. A hernia policy must include clinical guidelines, availability of LA and inexpensive, high-quality surgical mesh. A system for monitoring and evaluation of the quality of the surgical services delivered is also needed.
Almost $16 \%$ of the patients operated for groin hernia were female which is less than the $24 \%$ demonstrated in a recent but smaller study in Eastern Uganda, but more than twice the figures from other countries in Sub-Saharan Africa and Sweden $[6,23,32,33]$. This may be a result of a higher prevalence of groin hernia in Ugandan women compared to elsewhere, or differences in health seeking behavior. Further on, almost a quarter of the patients were children. Due to the high risk of incarceration in women and children, groin hernia is an absolute indication for surgery.

The strength of this study is that it is based on interviews with surgeons and on review of theater records from primary, secondary and tertiary hospitals throughout Uganda. This gives high internal validity. The weaknesses are as follows: not all GHs and lower level healthcare units where hernia repair is performed were included in the study. This was due to limited funding and time. Accessibility and quality of data in the theater records was an issue of concern in this study. Handwritten records are difficult to read and deteriorate over time. In some instances, hernia data were missing due to destruction or loss of records. The available data were imputed to cater for the missing data. Processing those records into meaningful information is time consuming. The study was carried out for the years 2013 and 2014. There is no groin hernia surgery initiative so far in Uganda, and hernia mesh is still not widely available. It is expected that the situation has not changed much since 2013. Outcomes of the groin hernia operations are one of the indicators for quality of care but were beyond the scope of this study.

Future research should focus on how to design largescale hernia surgery training initiatives and determining the epidemiology of groin hernia in women and children so that surgical services can be adapted to meet the specific needs of these groups. A hernia registry would facilitate the improvement transition and enable the making of timely reports and high-quality research in the future.

Acknowledgements Open access funding provided by Karolinska Institute. We acknowledge all those that supported this study, the directors and superintendents of the hospitals who gave us permission to collect the data, the staff who helped us locate the theater registers, the data collectors and the data entrants. We acknowledge the study participants that responded to our data collection tool.

Funding The study was funded through a grant from the Department of Public Health and Clinical Medicine, Unit of Research, Education, and Development, Östersund, Umeå University.

\section{Compliance with ethical standards}

Conflict of interest All authors declare that they have no conflict of interest. 
Open Access This article is licensed under a Creative Commons Attribution 4.0 International License, which permits use, sharing, adaptation, distribution and reproduction in any medium or format, as long as you give appropriate credit to the original author(s) and the source, provide a link to the Creative Commons licence, and indicate if changes were made. The images or other third party material in this article are included in the article's Creative Commons licence, unless indicated otherwise in a credit line to the material. If material is not included in the article's Creative Commons licence and your intended use is not permitted by statutory regulation or exceeds the permitted use, you will need to obtain permission directly from the copyright holder. To view a copy of this licence, visit http://creativecommons. org/licenses/by/4.0/.

\section{References}

1. Bay-Nielsen M, Kehlet H, Strand L, Malmstrøm J, Andersen FH, Wara P et al (2001) Quality assessment of 26,304 herniorrhaphies in Denmark: a prospective nationwide study. Lancet 358(9288): 1124-1128

2. Beard JH, Ohene-Yeboah M, Devries CR, Schecter WP (2015) Hernia and hydrocele. In: Debas HT, Donkor P, Gawande A, Jamison DT, Kruk ME, Mock CN (eds) Essential surgery: disease control priorities, vol 1, 3rd edn. The International Bank for Reconstruction and Development/The World Bank, Washington, DC

3. Kingsnorth AN, Clarke MG, Shillcutt SD (2009) Public health and policy issues of Hernia surgery in Africa. World J Surg 33(6):1188-1193. https://doi.org/10.1007/s00268-009-9964-y

4. Galukande M, von Schreeb J, Wladis A, Mbembati N, de Miranda H, Kruk ME et al (2010) Essential surgery at the district hospital: a retrospective descriptive analysis in three African countries. PLoS Med 7(3):e1000243

5. Ohene-Yeboah M, Beard JH, Frimpong-Twumasi B, Koranteng A, Mensah S (2016) Prevalence of inguinal hernia in adult men in the Ashanti region of Ghana. World J Surg 40(4):806-812. https://doi.org/10.1007/s00268-015-3335-7

6. Löfgren J, Makumbi F, Galiwango E, Nordin P, Ibingira C, Forsberg BC et al (2014) Prevalence of treated and untreated groin hernia in eastern Uganda. Br J Surg 101(6):728-734

7. Löfgren J (2015) Groin hernias and unmet need for surgery in Uganda epidemiology, mosquito nets and cost-effectiveness. Department of Surgical and Perioperative Sciences, Umeå University, Umeå

8. Grimes CE, Law RSL, Borgstein ES, Mkandawire NC, Lavy CBD (2012) Systematic review of met and unmet need of surgical disease in rural sub-Saharan Africa. World J Surg 36(1):8-23. https://doi.org/10.1007/s00268-011-1330-1

9. Beard JH, Oresanya LB, Ohene-Yeboah M, Dicker RA, Harris HW (2013) Characterizing the global burden of surgical disease: a method to estimate inguinal hernia epidemiology in Ghana. World J Surg 37(3):498-503. https://doi.org/10.1007/s00268-012-1864-x

10. Patel HD, Groen RS, Kamara TB, Samai M, Farahzad MM, Cassidy LD et al (2014) An estimate of hernia prevalence in Sierra Leone from a nationwide community survey. Hernia 18(2):297-303

11. Sachs M, Damm M, Encke A (1997) Historical evolution of inguinal hernia repair. World J Surg 21(2):218-223. https://doi. org/10.1007/s002689900220

12. Lichtenstein IL, Shulman AG, Amid PK, Montllor MM (1989) The tension-free hernioplasty. Am J Surg 157(2):188-193

13. Paajanen H, Varjo R (2010) Ten-year audit of Lichtenstein hernioplasty under local anaesthesia performed by surgical residents. BMC Surg. https://doi.org/10.1186/1471-2482-10-24
14. The HerniaSurge Group (2018) International guidelines for groin hernia management. Hernia 22(1):1-165

15. O'Dwyer PJ, Serpell MG, Millar K, Paterson C, Young D, Hair A et al (2003) Local or general anesthesia for open hernia repair: a randomized trial. Ann Surg 237(4):574-579

16. Amato B, Compagna R, Della Corte GA, Martino G, Bianco T, Coretti G et al (2012) Feasibility of inguinal hernioplasty under local anaesthesia in elderly patients. BMC Surg 12(Suppl 1):S2

17. Sanjay P, Woodward A (2007) Inguinal hernia repair: local or general anaesthesia? Ann Roy Coll Surg Engl 89(5):497-503

18. Dhumale R, Tisdale J, Barwell N (2010) Over a thousand ambulatory hernia repairs in a primary care setting. Ann Roy Coll Surg Engl 92(2):127-130

19. Saia M, Mantoan D, Buja A, Bertoncello C, Baldovin T, Zanardo $C$ et al (2013) Increased rate of day surgery use for inguinal and femoral hernia repair in a decade of hospital admissions in the Veneto Region (north-east Italy): a record linkage study. BMC Health Serv Res. https://doi.org/10.1186/1472-6963-13-349

20. The World Bank. Population, total [Internet]. The World Bank Data. Available from http://data.worldbank.org/indicator/SP. POP.TOTL. Accessed 26 Jan 2016

21. Katende D, Mutungi G, Baisley K, Biraro S, Ikoona E, Peck R et al (2015) Readiness of Ugandan health services for the management of outpatients with chronic diseases. Trop Med Int Health 20(10):1385-1395

22. Scott NW, McCormack K, Graham P, Go PM, Ross SJ, Grant AM (2002) Open mesh versus non-mesh for repair of femoral and inguinal hernia. Cochrane Database Syst Rev 4:CD002197

23. Nationella registergruppen för Svenskt Bråckregister. Registret för ljumskbråcksoperationer, Nationell rapport 2016

24. Simons MP, Aufenacker T, Bay-Nielsen M, Bouillot JL, Campanelli G, Conze J et al (2009) European Hernia Society guidelines on the treatment of inguinal hernia in adult patients. Hernia 13(4):343-403

25. Nordin P, Zetterström H, Gunnarsson U, Nilsson E (2003) Local, regional, or general anaesthesia in groin hernia repair: multicentre randomised trial. Lancet 362(9387):853-858

26. Nordin P, Zetterstrrm H, Carlsson P, Nilsson E (2007) Costeffectiveness analysis of local, regional and general anaesthesia for inguinal hernia repair using data from a randomized clinical trial. Br J Surg 94(4):500-505

27. Mabula JB, Chalya PL (2012) Surgical management of inguinal hernias at Bugando Medical Centre in northwestern Tanzania: our experiences in a resource-limited setting. BMC Res Notes 5(1):585

28. Devlin H (1995) Trends in hernia surgery in the land of Astley Cooper. In: Sooper NJ (ed) Problems in general surgery, 6th edn. Lippincott-Raven, Philadelphia, pp 85-92

29. Rutkow IM (1998) Epidemiologic, economic, and sociologic aspects of hernia surgery in the United States in the 1990s. Surg Clin North Am 78(6):941-951

30. Chu K, Rosseel P, Gielis P, Ford N (2009) Surgical task shifting in sub-Saharan Africa. PLoS Med 6(5):e1000078

31. Fulton BD, Scheffler RM, Sparkes SP, Auh EY, Vujicic M, Soucat A (2011) Health workforce skill mix and task shifting in low income countries: a review of recent evidence. Hum Resour Health 9(1):1

32. Mbah N (2007) Morbidity and mortality associated with inguinal hernia in Northwestern Nigeria. West Afr J Med 26(4):288-292

33. Arowolo OA, Agbakwuru EA, Adisa AO, Lawal OO, Ibrahim $\mathrm{MH}$, Afolabi AI (2011) Evaluation of tension-free mesh inguinal hernia repair in Nigeria: a preliminary report. West Afr J Med 30(2):110-113

Publisher's Note Springer Nature remains neutral with regard to jurisdictional claims in published maps and institutional affiliations. 